\title{
The Effect of City Bus Maneuvers on Wheelchair Movement
}

\author{
Michael J. Turkovich, Linda van Roosmalen, \\ Douglas A. Hobson, and Erik A. Porach \\ University of Pittsburgh
}

\begin{abstract}
A state-of-the-art four-point tiedown system, a prototype automatic docking system, and a prototype rear-facing wheelchair passenger station (RF-WPS) were installed in a large accessible transit vehicle (LATV). A manual wheelchair, powered wheelchair, and a three-wheeled scooter were used to test the securement performance of each wheelchair securement system during LATV normal driving, hard braking, and rapid turning maneuvers. All test wheelchairs were loaded with an ISO 7176 Part 11 compliant loader gage representing the weight of an average male wheelchair occupant. A tri-axial accelerometer measured vehicle acceleration during driving maneuvers, and a low-tech movement tracking system measured wheelchair movement during driving maneuvers. Results show that each wheelchair securement system limited wheelchair displacement to less than the 51- $\mathrm{mm}$ Americans with Disabilities Act (ADA) displacement limitation, and none of the securement systems showed visible signs of failure. Accelerations during LATV normal driving, hard braking, and rapid turning did not exceed $0.76 \mathrm{~g}$.
\end{abstract}

\section{Introduction}

\section{Regulations and Standards}

With the passage of the 1990 Americans with Disabilities Act (ADA), public transportation is required to be available and accessible to people with disabilities. The 
U. S. Department of Transportation (DOT) 49 CFR Part 38 requires public buses or large accessible transit vehicles (LATVs) to be outfitted with a wheelchair station consisting of a wheelchair securement and an occupant restraint system (U.S. Department of Transportation 2007). This regulation requires that the wheelchair securement system limit the movement of a wheelchair to a maximum of $51 \mathrm{~mm}$ (2 in.) during normal vehicle operating conditions. Although the ADA does not require wheelchairs to be secured, vehicle operators are to be trained to use the safety equipment on board public transit vehicles, and transit providers may have written policies in place requiring wheelchairs to be secured in the best possible way with the available equipment (U.S. Department of Transportation 2007).

Despite the advancement in legislation regarding accessible public transportation and the development of standards and compliant wheelchair transportation safety technology, vehicle operators and wheelchair users of public transportation systems have been reporting difficulties (Buning et al. 2007; Frost and Bertocci 2009). Lack of use of wheelchair securement systems has been attributed to the fact that many wheelchairs are difficult to secure. Buning et al. (2007) surveyed public transit wheelchair users and reported that over 50 percent had difficulty securing their wheelchairs. Lack of securement use also can be attributed to a lack of bus driver training in the proper use of wheelchair securement systems and a lack of compatible wheelchair securement hardware and wheelchair securement systems (Foreman et al. 2001). Currently, the most common type of wheelchair securement system installed in public buses is the four-point, strap-type tiedown system (Wolf and van Roosmalen 2007) due to the system's ability to accommodate a wide range of wheelchair types and sizes. A shortcoming of this securement system often cited by wheelchair users is that they have to rely on someone else to secure their wheelchair, thus not allowing independent use of the system. In addition, the bus driver has to secure the tiedown straps in hard-to-reach places on the wheelchair due to non-WC-19-compliant wheelchairs, which often impose on the user's personal space, further increasing the likelihood that wheelchair users will refuse the use of securement systems (Buning et al. 2007).

Voluntary standards that include design and performance requirements of wheelchair tiedowns and occupant restraint systems (WTORS) have been developed to improve the safety and ease of use of wheelchair transportation safety technology (ANSI/RESNA 2001; International Standards Organization 2001). Standards also are being developed for WTORS that will be used only in LATVs, such as the draft international standard (ISO-10865-1) on rear-facing wheelchair passenger systems 
(RF-WPSs) for use in low-g environments (International Standards Organization 2010). The purpose of ISO-10865-1 (which at the time of this writing is a Draft In Standard [DIS]) is to establish design and performance requirements for RF-WPS in a low-g environment $(<1 \mathrm{~g})$ such as in LATVs. The standard specifies dimensional, design, performance, and installation requirements for an RF-WPS and its components. In addition, guidelines are provided for use by vehicle and/or WTORS manufacturers seeking to design RF-WPS components (International Standards Organization 2010). This standard is intended to promote the development and implementation of alternative wheelchair transportation safety systems that can be used independently by wheelchair-seated passengers of LATVs.

\section{Development of Alternative Wheelchair Securement}

Earlier attempts have been made to increase the usability and independent use of wheelchair securement systems. Several alternative securement devices have been developed, including an automated docking system created by Oregon State in the 1990s and, more recently, an auto-docking system with a Universal Docking Interface Geometry (UDIG) developed and tested by the University of Pittsburgh (UPITT) and Sure-Lok. The UPITT/Sure-Lok system can be used independently by a wheelchair user and incorporates an anti-rotation lock to ensure that once the wheelchair is secured, the docking system will not rotate during rapid turning (Hobson and van Roosmalen 2007). This system was tested successfully according to SAE J2249 test methods with a surrogate wheelchair and a $48 \mathrm{kph} / 20 \mathrm{~g}$ crash pulse (Society of Automotive Engineers 1999). User testing of the auto docking system resulted in positive responses from wheelchair users and bus drivers on its ease of use (Hobson and van Roosmalen 2007).

RF-WPSs also have been developed for independent use by wheelchair-seated occupants using LATVs in Europe, Canada, and the U.S. (Rutenberg et al. 2005). RFWPSs use a forward excursion barrier (FEB) that prevents the wheelchair and occupant from moving forward in the event of a sudden stop. Although some of these rear-facing systems include optional restraint systems that attach to or around the wheelchair, it remains unclear what level of containment is needed to protect the wheelchair and occupant from moving and/or tipping over during rapid vehicle turns and accelerations.

\section{LATV Accelerations}

Several studies have analyzed the accelerations that are experienced on board LATVs during various driving maneuvers. Hunter-Zaworski et al. (1992) measured in-vehicle accelerations during normal driving operations of LATVs. Their results 
indicate that during normal driving conditions, maximum accelerations reach $0.40 \mathrm{~g}$ and $0.10 \mathrm{~g}$ for forward acceleration and turning, respectively (Zaworski et al. 2007). Rutenberg (1995) reported that accelerations did not exceed $0.24 \mathrm{~g}$ in any direction (Rutenberg 1995). Fournier (1997) measured accelerations on LATVs as well and concluded that accelerations can be as high as $1.53 \mathrm{~g}$, but it was suggested that the high values seen were likely due to vibrations of the vehicle (Fournier 1997). Finally, Zaworski et al. (2007) recorded normal driving and extreme driving accelerations of LATVs and found that during normal driving conditions, accelerations averaged $0.20 \mathrm{~g}$ and rarely exceeded $0.40 \mathrm{~g}$. During extreme maneuvers, vehicle accelerations averaged $0.40 \mathrm{~g}$ and sometimes reached as high as $0.80 \mathrm{~g}$ during hard stops (Zaworski et al. 2007).

The combination of large vehicle size and relatively low travel speeds leads to a low likelihood of LATVs being involved in a collision of significant magnitude (Shaw and Gillispie 2003; Shaw 2008). Blower et al. (2005) examined accident reports from LATVs in Florida over a two-year period. They estimated that LATVs are involved in a collision of greater than $5 \mathrm{~g}$ every 27 million vehicle-miles traveled and in a $10 \mathrm{~g}$ collision every 455 million vehicle miles traveled. The likelihood of an LATV being involved in a crash event of 5 and $10 \mathrm{~g}$ is 16 and 250 times less, respectively, than for private vehicles (Blower et al. 2005). Given this low likelihood of a severe crash, the low $g$ environment of LATVs, and the large size of LATVs, alternative wheelchair containment methods may offer a reasonable level of occupant safety to wheelchair users traveling in LATVs while allowing for greater freedom in the design of these systems and for designs that promote independent use by wheelchair-seated passengers.

A $20 \mathrm{~g} / 48 \mathrm{kph}(20 \mathrm{~g} / 30 \mathrm{mph})$ frontal impact test with a surrogate wheelchair and Hybrid III Anthropomorphic Test Device is commonly used to evaluate wheelchair securement system safety. Alternative test methods that evaluate wheelchair securement system safety and assess wheelchair movement under lower accelerations commonly seen in LATVs are needed to improve wheelchair-seated passenger safety during normal driving conditions.

\section{Objectives}

The primary goal of this study was to determine if a prototype auto-docking system and a prototype RF-WPS are compliant with ADA maximum displacement requirements of $51 \mathrm{~mm}$ (2-in.) when exposed to accelerations associated with LATV normal driving, hard braking, and rapid turning. Secondary goals were to evaluate wheelchair securement performance by comparing the wheelchair displacement allowed by the two prototype systems with the displacement allowed 
by a standard four-point tiedown securement and no wheelchair securement. A final goal was to document the magnitude of LATV accelerations during normal driving, hard braking, and rapid turning conditions.

A follow-up project to this study includes having wheelchair users and LATV operators use and evaluate each wheelchair securement system on an LATV over a predetermined route and give feedback as to their likes and dislikes of each system. Findings of these wheelchair user and bus operator perceptions on wheelchair securement usage are being published separately.

\section{Methods}

Three types of wheelchair securement systems were installed in a high-floor LATV. Each wheelchair securement system was tested with a manual wheelchair, a powered wheelchair, and a scooter that were slightly modified to accommodate the securement systems tested (modifications of the test wheelchairs are described later on in the Methods section). The wheelchair securement systems were evaluated under three driving conditions: normal driving, hard braking, and rapid turning. The wheelchairs also were tested under each driving condition without any form of wheelchair securement. Wheelchair displacement and LATV accelerations were recorded during all testing conditions.

\section{Wheelchair Securement Setup}

\section{Four-Point Tiedown System}

The four-point tiedown system that was installed in the LATV consists of four straps with self-tensioning retractors (QRT Deluxe Retractor System, Q'Straint, Ft. Lauderdale). The straps have securement hook end fittings that each attach to four securement points on the wheelchair. The retractors contain a manual tension mechanism that allows a person to tighten the straps to minimize wheelchair movement. Two straps are secured to the rear and two to the front of the wheelchair (Figure 1). 

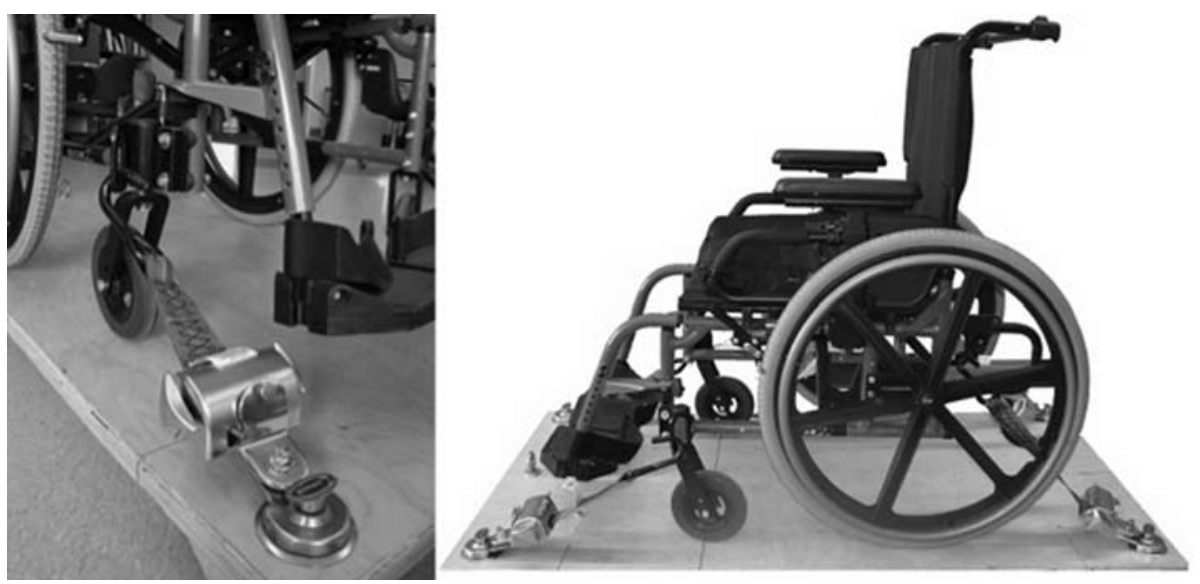

Figure 1. Retractor-type four-point tiedown system (left); side view of manual wheelchair secured by four-point tiedown system (right)

\section{Auto Docking System}

The prototype forward-facing auto docking system that was installed in the LATV was developed by the University of Pittsburgh and Sure-Lok (Sure-Lok, Bethlehem, PA). It consists of a pneumatically-powered docking mechanism that engages with an UDIG adaptor on the rear frame of each wheelchair (Figure 2). When a wheelchair user backs into the auto-docking system, the UDIG provides a means for securing the wheelchair to the docking securement device (Figure 3).
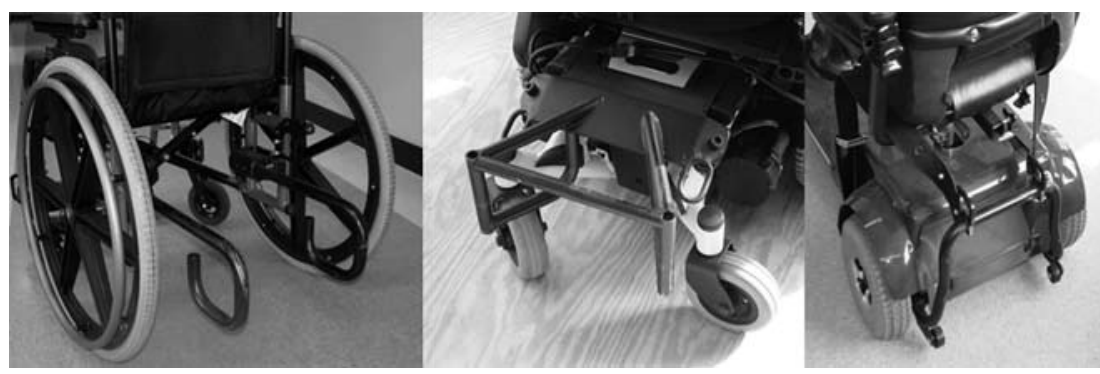

Figure 2. UDIGs attached to manual wheelchair (left), powered wheelchair (middle), and scooter (right) 


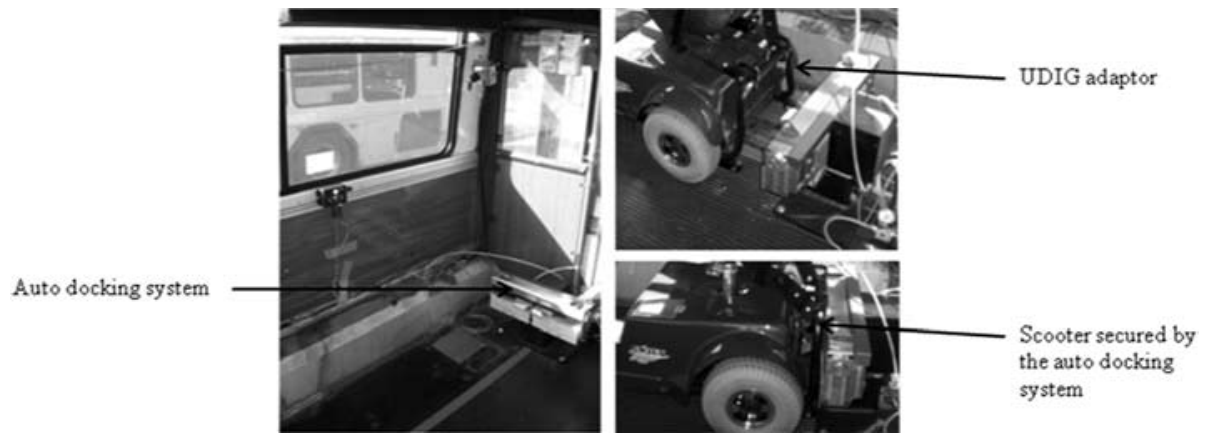

Figure 3. Auto docking system installed in LATV (left); scooter equipped with UDIG adaptor, backed into and secured by auto docking system

\section{RF-WPS System}

A prototype RF-WPS developed by the University of Pittsburgh and Q'Straint was installed in the LATV (Figure 4). This system consists of a vehicle-anchored FEB and two pneumatically activated lateral barriers. On the aisle side, the lateral barrier consists of a padded arm that rotates from a vertical (downward) stored position to an in-use (45-degree) position, while on the wall side the lateral barrier consists of a padded movable block. The pneumatic lateral barriers move laterally to accommodate different wheelchair positions and widths. These lateral barriers squeeze the sides of a wheelchair to provide containment and to prevent lateral and rearward movement of the wheelchairs during low $g$ non-crash accelerations of an LATV. The RF-WPS system does not require wheelchair-mounted hardware (e.g., UDIG adaptor), and the system does not include an occupant restraint system.

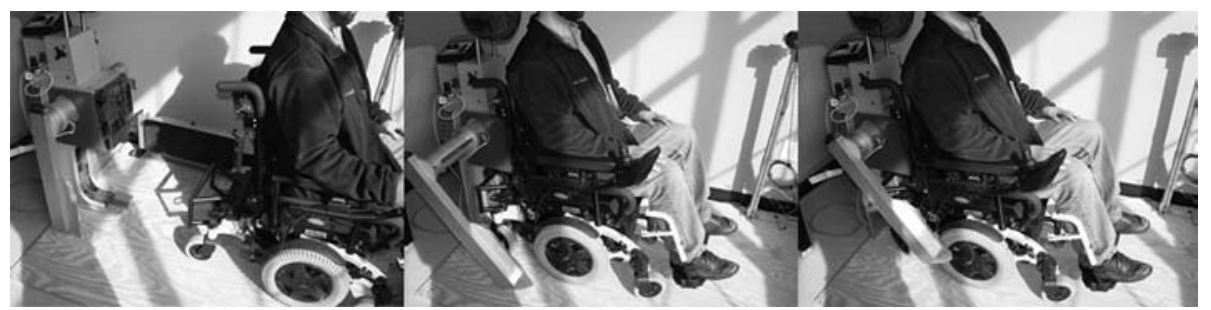

Figure 4. Rear-facing wheelchair passenger station (RF-WPS)

\section{Test Wheelchair Setup}

Three commonly-used wheeled mobility devices were selected for the purpose of examining the effectiveness of the three wheelchair securement systems. Each test wheelchair was equipped with four tiedown securement points (to work with the four-point tiedown system), a UDIG adaptor (to work with the auto docking 
system), and a wheelchair-anchored pelvic belt (to restrain the loader gages in each wheelchair). Pelvic belt prototypes were provided by BodyPoint (BodyPoint, Seattle) and Q'Straint. Each wheelchair was loaded with an ISO 7176-11 (International Standards Organization 1992), 76 kg (168 lbs) loader gage, representing a 50th percentile male occupant.

The three test wheelchairs included in the study were a manual wheelchair, a powered wheelchair, and a three-wheeled scooter. An ISO 7176-19 compliant Quickie II manual wheelchair (Sunrise Medical, Longmont, CO) had WC19 compliant securement points and was modified to include a prototype UDIG adaptor and a wheelchair-anchored pelvic restraint (Figure 5a). A WC19 compliant Invacare TDX-SP powered wheelchair (Invacare, Cleveland) was modified with a prototype UDIG adaptor wheelchair (Figure 5b). An Amigo-RD scooter (Amigo Mobility International, Bridgeport, $\mathrm{MI}$ ) was modified with four tiedown securement points, a prototype UDIG adaptor, and a UDIG-mounted pelvic restraint (Figure $5 \mathrm{c}$ ). The securement points on the scooter were designed and placed to allow for easy and effective securement by the bus operator with the four-point tiedown system. Although the securement point geometry on the scooter complied with WC19 dimensional requirements, the securement point locations on the scooter were not in compliance with the fore-aft and side-side WC19 requirements due to restrictions of suitable scooter frame mounting positions (ANSI/RESNA 2001).
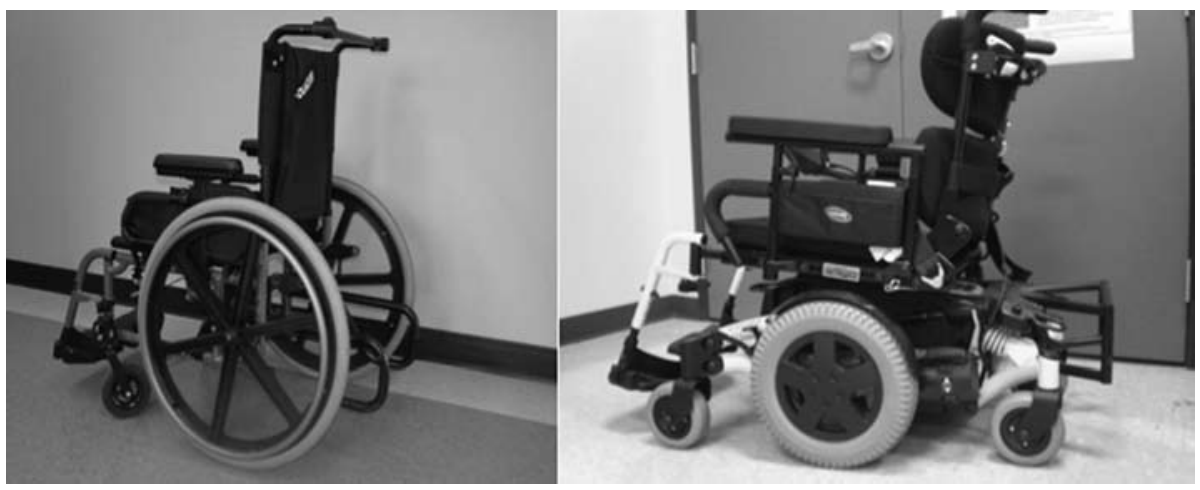

Figure 5. (a) Modified Quickie II manual wheelchair, (b) Invacare TDX-SP powered wheelchair, (c) Amigo-RD Scooter with ISO 7176-11 Test Dummy

\section{In-Vehicle Test Setup}

The Pittsburgh Port Authority (PAT) provided a $12.2 \mathrm{~m}$ (40 ft) transit bus (ORION Bus Industries Inc., Oriskany, NY) and a licensed PAT bus driver for testing pur- 
poses. The test vehicle was a high-floor vehicle without internal wheel wells. The test vehicle had a front mounted platform lift, and some seats were removed from the vehicle to allow for installation of the three wheelchair securement systems to be evaluated. The four-point tiedown system was placed in the row behind the driver seat and installed according to Q'Straint WTORS installation instructions. The prototype automated docking system and prototype RF-WPS were placed opposite each other on the right side of the vehicle. Figure 6 shows a diagram of the securement system setup in the LATV.

Front of LATV

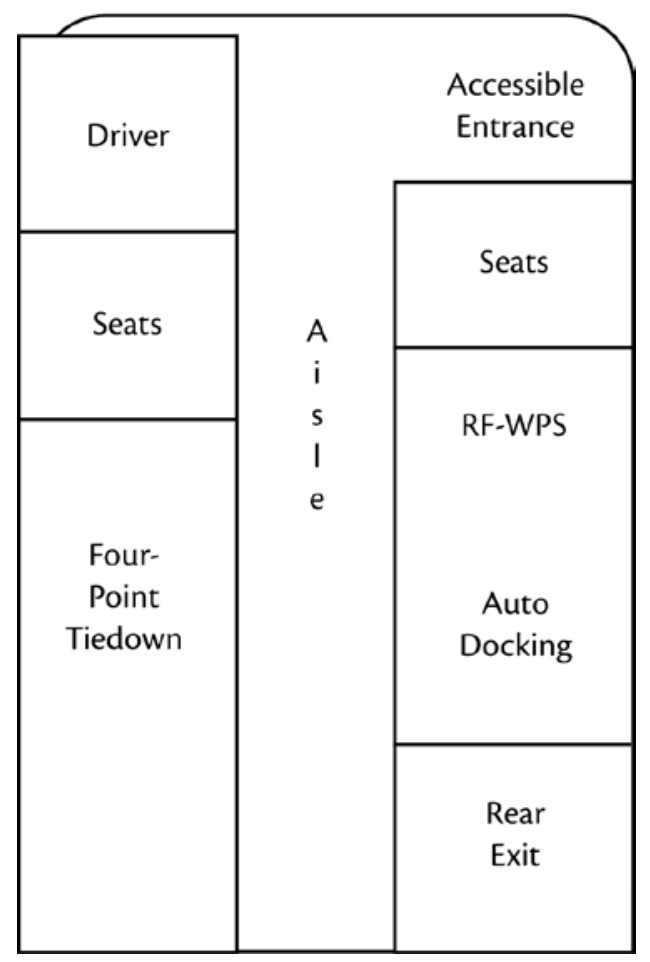

Figure 6. Layout of securement systems in LATV

\section{Driving Conditions}

Each securement system was tested under three driving conditions: normal driving, hard braking, and rapid turning. An urban course approximately 15 minutes in duration was mapped out for use during normal driving conditions. The course consisted of multiple left and right turns, starts, stops, and steep inclines and declines with a maximum grade of 17 percent. Hard braking trials are defined by LATV 
braking at a starting speed of about $32 \mathrm{~km} / \mathrm{h}(20 \mathrm{mph})$ to an end speed of $0 \mathrm{~km} / \mathrm{h}$ $(0 \mathrm{mph})$ in approximately 3 seconds. Rapid turning trials are defined by a 90 degree left or right turn at a starting speed of about $32 \mathrm{~km} / \mathrm{h}(20 \mathrm{mph})$ along a marked 15 meter (50 ft.) radius (Mercer and Billing 1990; Hobson and van Roosmalen 2007). LATV speeds and actual paths were not documented during the test trials.

\section{Test Protocol}

The three test wheelchairs were evaluated in three securement stations during three driving trials (normal driving, hard braking/ rapid turning, no securement):

- Three trials were conducted for the normal driving condition. Each wheelchair was tested on this course in each securement system to understand securement system performance for each wheelchair type during normal driving conditions.

- Eighteen trials of hard braking and rapid turning testing were conducted. Each wheelchair was tested three times in each station for both hard braking and rapid turning. A hard braking test was performed during the initial positioning of the wheelchairs to make sure the setup was appropriate. Thus, there were some cases where the systems were tested three times and others four.

- Wheelchairs also were tested when unsecured during normal driving, hard braking and rapid turning. Wheelchairs were unsecured with the hand brakes on (manual wheelchair) and power off (powered wheelchair and scooter). The wheelchairs and loader gages were loosely tethered to the vehicle walls by ropes to prevent excessive movement of and damage to the wheelchairs and loader gages.

Data were collected for a total of 28 trials. Throughout the testing, maximum vehicle accelerations and maximum wheelchair displacement were recorded for each wheelchair. A stationary video camera was used to observe general wheelchair motion.

\section{Data Collection and Analysis}

To capture wheelchair displacement, a low-cost, previously-validated test method was used (Hobson and van Roosmalen 2007). A target designed to contain a $51 \mathrm{~mm}$ ( 2 in.) radius circle, representative of the ADA displacement requirement, was fixed to the vehicle floor. The ADA does not specify how or where the displacement of a wheelchair should be measured from, so for the purposes of this study a springloaded pen was attached to the front of the wheelchair frame at the centerline of each wheelchair and $780 \mathrm{~mm}$ (30.7 in.), $830 \mathrm{~mm}$ (32.7 in.), and $1110 \mathrm{~mm}$ (43.7 in.) 
forward of the vertical securement bars of the UDIG, for the manual wheelchair, powered wheelchair, and scooter respectively. Prior to the start of each trial, the pen was positioned at the center of the $51 \mathrm{~mm}(2 \mathrm{in}$.) radius target so that any movement from the original position would scribe a line that could be measured post-test (Figure 7). The displacement magnitude $(\mathrm{mm})$ was measured from each of the marked targets located beneath each wheelchair. The furthest deviation from the target center was recorded as the maximum displacement. Displacement was measured to the nearest $\mathrm{mm}$. If wheelchair displacement exceeded the width/ length of the chart-108 mm (4 in.) in the lateral direction and $140 \mathrm{~mm}(5.5 \mathrm{in}$.) in the forward/rearward direction-it was labeled as "off the chart," or if the wheelchair tipped over, it was labeled as "tipped over."

Analysis of Variance (ANOVA) was used to determine statistical differences in displacement values of the test wheelchairs and scooter during various driving conditions when secured by each wheelchair securement system. An alpha level of 0.05 was used to determine significance. Additionally, maximum values of forward and lateral test wheelchair movement were measured and tabulated for the various driving conditions for each test wheelchair and each wheelchair securement scenario. Acceleration time histories were recorded for each test trial, and maximum LATV accelerations and average LATV accelerations were tabulated for normal driving, hard braking, and rapid turning conditions.

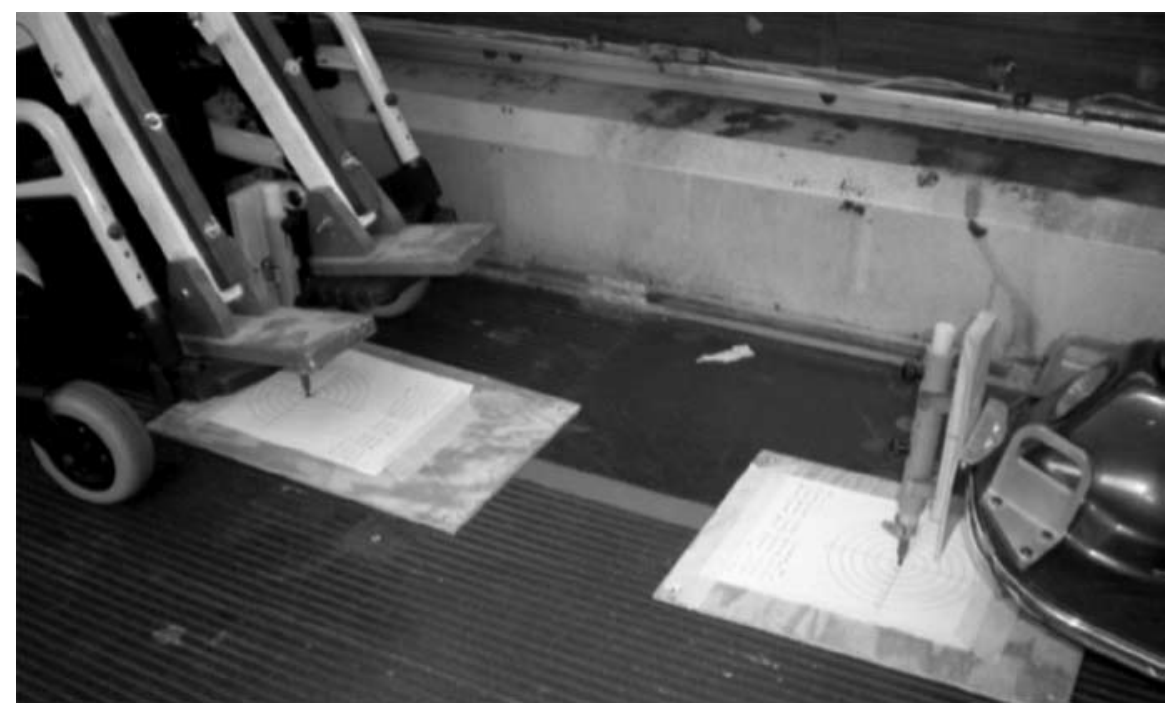

Figure 7. Spring-loaded pens and targets used for recording displacements of wheelchairs 
A $2.5 \mathrm{~g}$ tri-axial accelerometer (GP1 Sensr, Elkader, IA) was fixed to the floor of the bus and positioned so that the axes $(x, y, z)$ of the accelerometer were aligned with the longitudinal axis of the vehicle, the lateral horizontal axis of the vehicle, and the vertical, respectively. The accelerometer was positioned on the left side (driver side) of the bus, directly behind and in line with the center of the four-point tiedown station. Acceleration data were recorded for all trials at a frequency of $100 \mathrm{~Hz}$.

Accelerometer data were processed similar to that of Zaworski et al. (2007). The accelerometer data first were averaged to $20 \mathrm{~Hz}$, and voltage offsets were adjusted, and the raw voltage signal was converted to units of $g$ in accordance with SAE $J 2181$ (Society of Automotive Engineers, 1993). Maximum vehicle accelerations were obtained for normal driving, hard braking, and rapid turning maneuvers, and all accelerations were reported in units of "g." Then $x, y$ and resultant accelerations were computed and reported for hard braking, rapid-turning, and normal driving conditions, respectively.

\section{Results}

\section{Wheelchair Displacement during Normal Driving}

Table 2 shows the maximum wheelchair displacement values recorded for each securement system during normal driving conditions. During the normal driving trials, no extraordinary events such as "jumping curbs" took place, and the route did not include steep uphill and downhill slopes of more than 17 percent. The maximum displacement recorded during normal driving was $18 \mathrm{~mm}$ ( $0.7 \mathrm{in}$.). This displacement was recorded on the target beneath the scooter when it was secured by the four-point tiedown system. The average maximum displacement experienced by the three wheelchairs was $12 \mathrm{~mm}( \pm 4 \mathrm{~mm})(0.47$ in. $\pm 0.16 \mathrm{in}$.). Displacements measured during normal driving trials were not synchronized with LATV accelerations at which maximum wheelchair displacements occurred.

Table 2. Maximum Wheelchair Displacement For All Normal Driving Trials

\begin{tabular}{|l|c|c|c|}
\hline Wheelchair Type & RF-WPS $(\mathbf{m m})$ & $\begin{array}{c}\text { Four-Point } \\
\text { Tiedown }(\mathbf{m m})\end{array}$ & $\begin{array}{c}\text { Auto Docking } \\
(\mathbf{m m})\end{array}$ \\
\hline Manual & 13 & 13 & 14 \\
\hline Powered & 11 & 6 & 11 \\
\hline Scooter & 13 & 18 & 6 \\
\hline Average (SD) & $11.67(1.15)$ & $12.33(6)$ & $10.33(4)$ \\
\hline
\end{tabular}




\section{Wheelchair Displacement during Hard Braking}

Table 3 shows the wheelchair displacements and accelerations for the three securement systems during hard braking. The maximum displacement during hard braking was $44 \mathrm{~mm}$ (1.7 in.). This displacement value was recorded on the target beneath the scooter during hard braking when secured by the four-point tiedown system.

\section{Table 3. Maximum Wheelchair Displacement And Associated Vehicle Acceleration During Hard Braking}

\begin{tabular}{|c|c|c|c|c|c|c|}
\hline & \multicolumn{2}{|c|}{ RF-WPS } & \multicolumn{2}{|c|}{ Four-Point Tiedown } & \multicolumn{2}{|c|}{ Auto Docking } \\
\hline & $(m m)$ & $(g)$ & $(\mathrm{mm})$ & $(g)$ & $(\mathrm{mm})$ & $(g)$ \\
\hline \multicolumn{7}{|c|}{ Manual Wheelchair } \\
\hline Trial 1 & 0 & 0.62 & 0 & 0.60 & 0 & 0.62 \\
\hline Trial 2 & 6 & 0.72 & 0 & 0.66 & 0 & 0.69 \\
\hline Trial 3 & 0 & 0.68 & 0 & 0.56 & 0 & 0.56 \\
\hline Trial 4 & 0 & 0.68 & $\mathrm{n} / \mathrm{a}$ & $\mathrm{n} / \mathrm{a}$ & $\mathrm{n} / \mathrm{a}$ & $\mathrm{n} / \mathrm{a}$ \\
\hline \multicolumn{7}{|c|}{ Powered Wheelchair } \\
\hline Trial 1 & 19 & 0.60 & 13 & 0.62 & 0 & 0.62 \\
\hline Trial 2 & 13 & 0.66 & 13 & 0.69 & 0 & 0.72 \\
\hline Trial 3 & 13 & 0.56 & 13 & 0.56 & 0 & 0.68 \\
\hline Trial 4 & $\mathrm{n} / \mathrm{a}$ & $\mathrm{n} / \mathrm{a}$ & $\mathrm{n} / \mathrm{a}$ & $\mathrm{n} / \mathrm{a}$ & 0 & 0.68 \\
\hline \multicolumn{7}{|l|}{ Scooter } \\
\hline Trial 1 & 13 & 0.62 & 44 & 0.62 & 0 & 0.60 \\
\hline Trial 2 & 13 & 0.69 & 24 & 0.72 & 0 & 0.66 \\
\hline Trial 3 & 6 & 0.56 & 24 & 0.68 & 0 & 0.56 \\
\hline Trial 4 & $\mathrm{n} / \mathrm{a}$ & $\mathrm{n} / \mathrm{a}$ & 24 & 0.68 & $\mathrm{n} / \mathrm{a}$ & $\mathrm{n} / \mathrm{a}$ \\
\hline Average (SD) & $8.3(6.8)$ & $0.64(0.1)$ & $12(10)$ & $0.64(0.1)$ & $0(0)$ & $0.64(0.1)$ \\
\hline
\end{tabular}

\section{Wheelchair Displacement during Turning}

Table 4 shows the wheelchair displacements and accelerations for the three securement systems during turning trials. The powered wheelchair secured by the four-point tiedown system showed the largest displacement of $41 \mathrm{~mm}$ (1.6 in.). The four-point tiedown system also allowed $37 \mathrm{~mm}$ (1.5 in.) of displacement of the manual wheelchair. 
Table 4. Maximum Wheelchair Displacement And Associated Vehicle Acceleration During Rapid Right Turning

\begin{tabular}{|c|c|c|c|c|c|c|}
\hline \multirow{2}{*}{} & \multicolumn{2}{|c|}{ RF-WPS } & \multicolumn{2}{c|}{ Four-Point Tiedown } & \multicolumn{2}{c|}{ Auto Docking } \\
\cline { 2 - 7 } & $(\mathrm{mm})$ & $(g)$ & $(\mathrm{mm})$ & $(g)$ & $(\mathrm{mm})$ & $(g)$ \\
\hline Manual Wheelchair & \multicolumn{5}{|c|}{} \\
\hline Trial 1 & 13 & 0.46 & 37 & 0.47 & 13 & 0.38 \\
\hline Trial 2 & 13 & 0.51 & 25 & 0.46 & 18 & 0.52 \\
\hline Trial 3 & 13 & 0.56 & 19 & 0.45 & 13 & 0.45 \\
\hline Powered Wheelchair & \multicolumn{7}{|l|}{} & \\
\hline Trial 1 & 13 & 0.47 & 13 & 0.38 & 13 & 0.46 \\
\hline Trial 2 & 13 & 0.46 & 41 & 0.52 & 13 & 0.51 \\
\hline Trial 3 & 13 & 0.45 & 19 & 0.45 & 13 & 0.56 \\
\hline Scooter & & & & & \\
\hline Trial 1 & 8 & 0.38 & 0 & 0.46 & 8 & 0.47 \\
\hline Trial 2 & 13 & 0.52 & 0 & 0.51 & 6 & 0.46 \\
\hline Trial 3 & 13 & 0.45 & 0 & 0.56 & 6 & 0.45 \\
\hline Average (SD) & $12(1.7)$ & $0.47(0.1)$ & $17(16)$ & $0.47(0.1)$ & $11(4.0)$ & $0.47(0.1)$ \\
\hline
\end{tabular}

\section{Maximum Wheelchair Displacement}

Maximum displacement values of the test wheelchairs and scooter during various driving conditions (normal driving, hard braking and rapid turning) when secured by each wheelchair securement system are displayed in Figure 8. The auto-docking system allowed significantly less wheelchair displacement than the four-point tiedown system $(p=0.0004)$ over all driving conditions. The displacement allowed by the RF-WPS during all driving conditions was not significantly different from that of the four-point tiedown system ( $p=0.1178)$ and the auto-docking system $(p=0.105)$.

\section{Unsecured Wheelchair Displacement}

Table 5 shows the displacement of the three test wheelchairs when they were unsecured under normal driving, hard braking, and rapid turning conditions when facing forward and rearward. As expected, all wheelchairs experienced increased displacement when unsecured. During normal driving, the manual wheelchair moved a large enough distance that the excursion indicator was off the chart. This movement occurred during vehicle braking on a downhill grade of 17 percent. During a rapid right turn, the manual wheelchair tipped into the aisle until the safety rope stopped the movement, and the scooter tipped towards the vehicle wall and the dummy impacted and fractured a side window (Figure 9). 


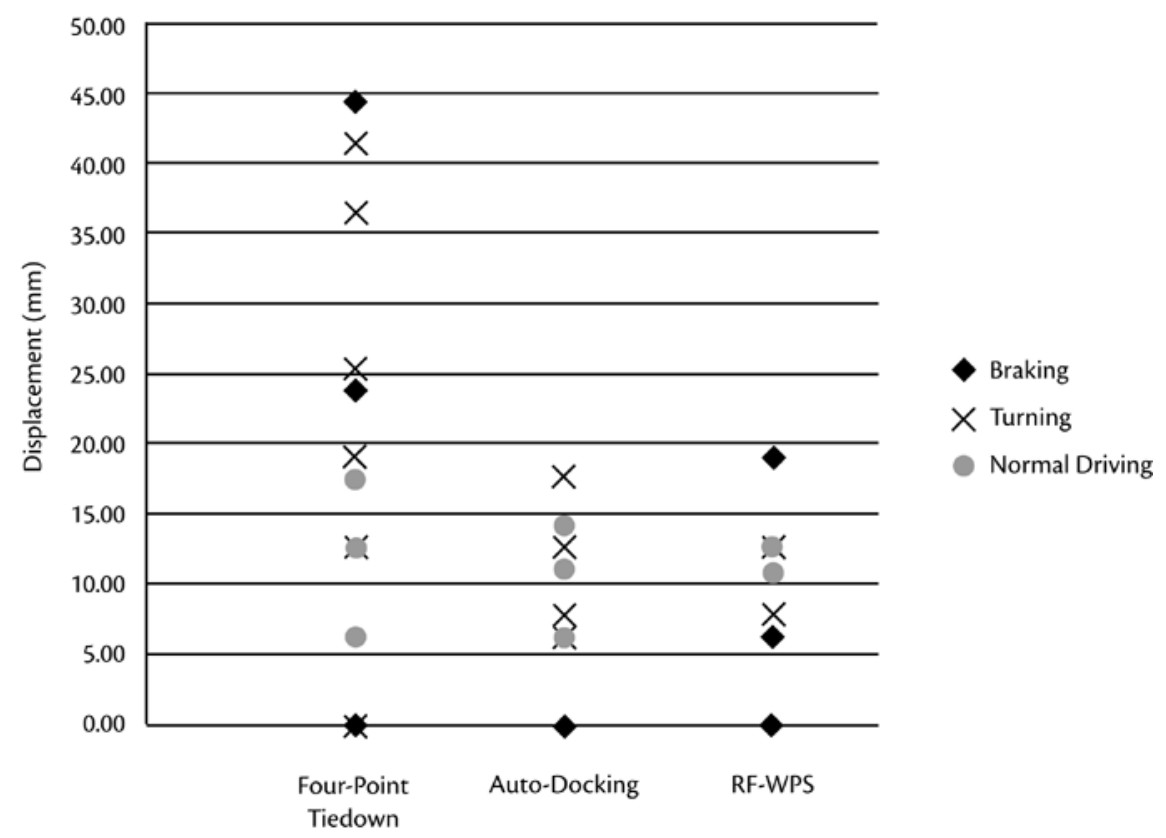

Figure 8. Maximum wheelchair displacement for four-point tiedown system, auto-docking system, and RF-WPS for test wheelchairs and scooter

\section{Table 5. Maximum wheelchair displacements for unsecured trials during normal driving, hard braking, and rapid turning}

\begin{tabular}{|c|c|c|c|}
\hline \multirow{2}{*}{ Hard Braking } & Manual & Powered & Scooter \\
\cline { 2 - 4 } & $\mathrm{mm}$ & $\mathrm{mm}$ & $\mathrm{mm}$ \\
\hline Forward Facing & \multicolumn{3}{|c|}{} \\
\hline Rear Facing & off the chart & off the chart & off the chart \\
\hline Rapid Turning & 0 & 13 & 19 \\
\hline Forward Facing & \multicolumn{3}{|c|}{} \\
\hline Rear Facing & off the chart & off the chart & tipped over \\
\hline Normal Driving & tipped over & 14 & off the chart \\
\hline Forward Facing & \multicolumn{3}{|c}{} \\
\hline Rear Facing & off the chart & 19 & 48 \\
\hline
\end{tabular}

Note: "Off the chart" indicates that the wheelchair moved sideways or forward outside the $51 \mathrm{~mm}$ (2 in.) target area and out of the wheelchair securement station, resulting in potential bodily injury to (wheelchair-seated) passengers. "Tipped over" indicates that the wheelchair or scooter tipped over sideways, resulting in potential bodily injury to (wheelchair-seated) passengers. 


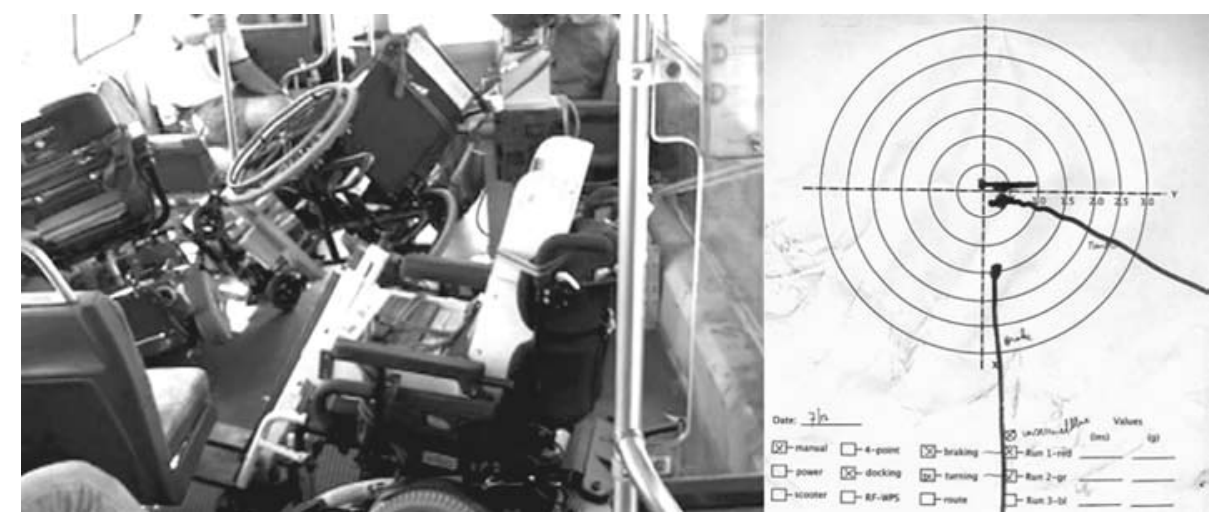

Figure 9. Final locations of unsecured wheelchairs during a right turn (left) and example of "off the chart" displacement (right)

\section{LATV Accelerations}

The maximum LATV accelerations were measured during normal driving, hard braking and rapid turning trials with the test wheelchairs and scooter. During normal driving conditions, the maximum acceleration measured was $0.60 \mathrm{~g}$. Since the normal driving trials were all approximately 15 minutes in length, time history curves displayed merely a flat line. The maximum acceleration measured during hard braking was $0.75 \mathrm{~g}$. Figure 10 shows the time history curves of all accelerations in the longitudinal ( $x$-axis) direction for hard braking. The maximum acceleration recorded during rapid turning was $0.56 \mathrm{~g}$. Figure 11 shows the time history curves of all accelerations in the lateral ( $y$-axis) direction for rapid turning. 


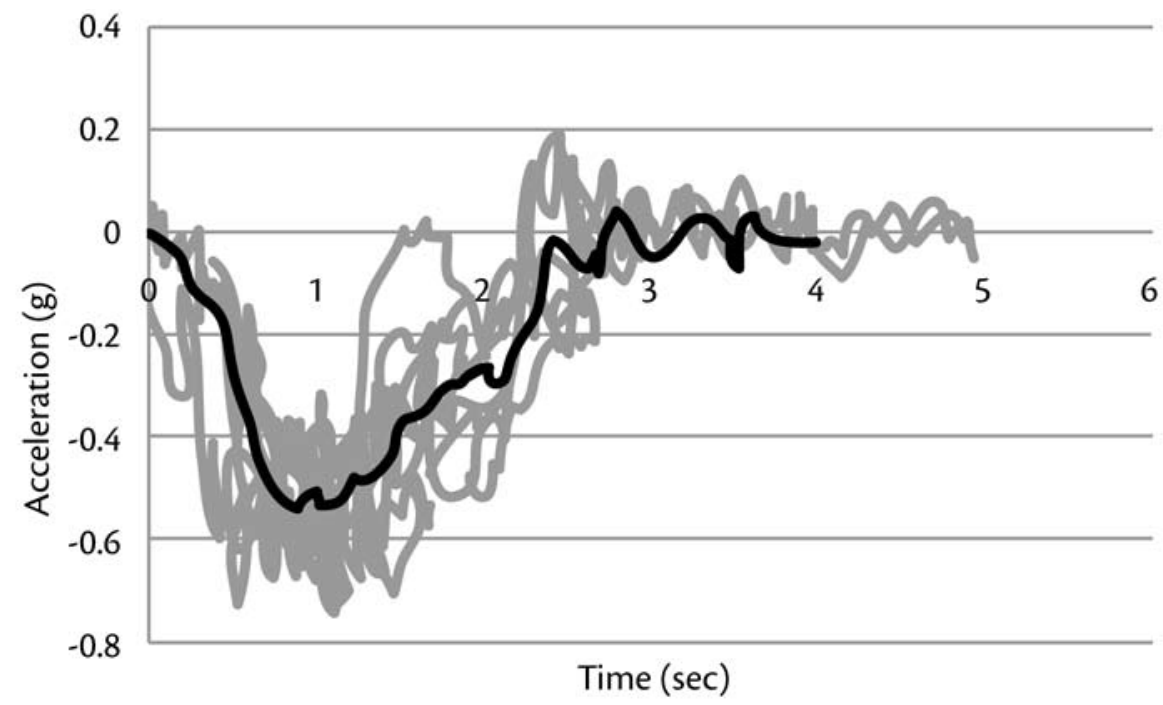

Figure 10. Time history acceleration curves in longitudinal ( $x$-axis) direction for $\mathbf{1 3}$ hard braking trials, and average time history acceleration curve (black line)

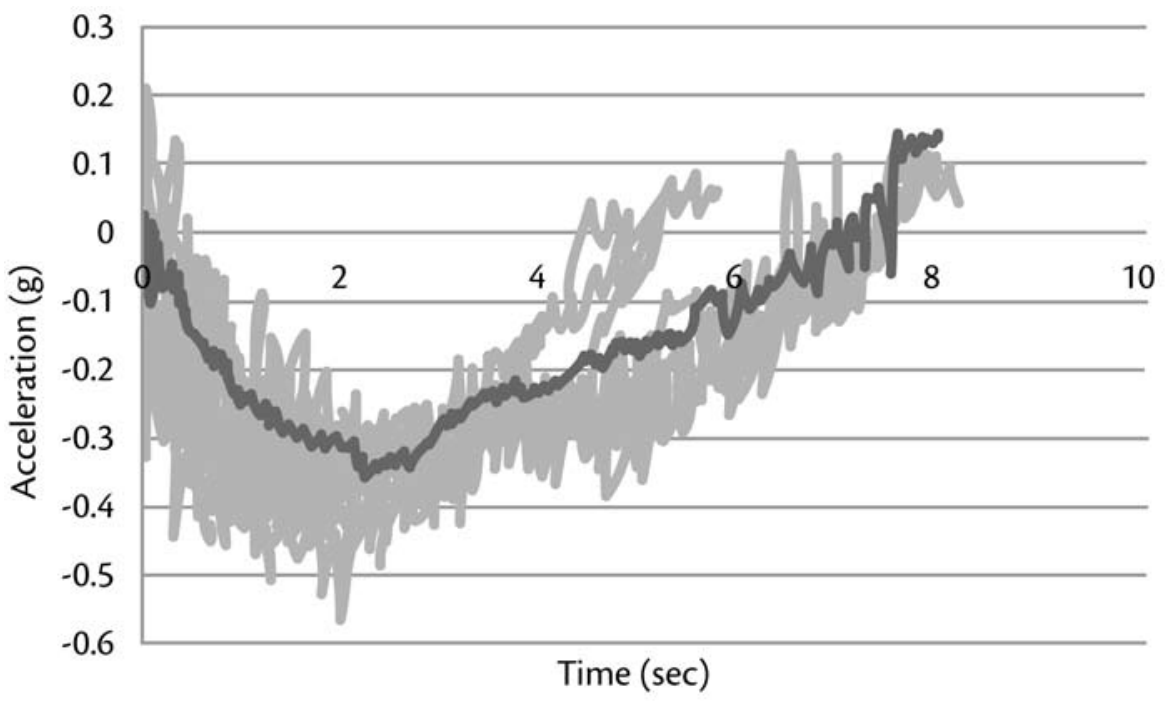

Figure 11. Time history acceleration curves in lateral (y-axis) direction for 12 rapid turning trials, and average time history acceleration curve (black line) 


\section{Discussion}

\section{Wheelchair Displacement}

Maximum wheelchair displacement measured on the test wheelchairs and the scooter that were secured by either a four-point tiedown system, a RF-WPS, or an auto-docking system were all within the maximum ADA allowed limit of $51 \mathrm{~mm}$ (2 in.). The highest displacement of $44 \mathrm{~mm}$ (1.7 in.) occurred with the scooter secured in the four-point tiedown system, followed by $19 \mathrm{~mm}(0.8 \mathrm{in}$.) with the powered wheelchair in the RF-WPS, and $18 \mathrm{~mm}(0.7 \mathrm{in}$.) with the manual wheelchair in the auto-docking system. Based on the $51 \mathrm{~mm}$ ( 2 in.) displacement criteria, all three systems would be ADA compliant. During normal driving, the maximum displacement was $18 \mathrm{~mm}$ ( $0.7 \mathrm{in}$.), and all systems had similar average displacements of $12 \mathrm{~mm}$ ( $0.5 \mathrm{in}$.), $12 \mathrm{~mm}$ ( $0.5 \mathrm{in}$.), and $10 \mathrm{~mm}$ (0.4 in.) for the RF-WPS, four-point tiedown system and auto-docking systems, respectively.

The auto-docking system performed best under hard braking conditions, allowing $0 \mathrm{~mm}$ ( $0 \mathrm{in}$.) of wheelchair displacement across wheelchair types. It allowed more movement during rapid turning conditions (average $11 \mathrm{~mm}$ [0.4in.]), but this is to be expected, as the UDIG interface to the docking system is located at the rear of each wheelchair. This rear anchoring arrangement would allow for the wheelchair's center of mass to rotate more in a turning than in a braking condition. The docking system allowed a maximum of $18 \mathrm{~mm}$ ( $0.7 \mathrm{in}$.) of lateral displacement, but this maximal displacement was well below the ADA maximum $51 \mathrm{~mm}$ (2 in.) displacement limit. Most of the variability in the movement allowed by an auto-docking system could possibly be attributed to the differences in pen location with respect to the UDIG, which was different for the test wheelchairs and scooter.

The RF-WPS design also was effective in retaining wheelchairs, as the maximum forward (powered) wheelchair displacement was $19 \mathrm{~mm}$ (0.8 in.), which occurred during hard braking. This is most likely due the fact that the padding on the FEB and wheel locks on wheelchairs allow for some movement of the (powered) wheelchair even when it is backed up against the FEB. The RF-WPS generally allowed more displacement during rapid turning (avg. $12 \mathrm{~mm}$ [0.5 in.]) than hard braking (avg. $8 \mathrm{~mm}[0.3 \mathrm{in}$.$] ) and there was no significant difference between the displace-$ ment allowed by the RF-WPS and the displacement allowed by the four-point tiedown system and auto-docking system. Wheelchairs contained by the RF-WPS prototype stayed within the ADA displacement limit of $51 \mathrm{~mm}$ ( 2 in.), thus the RFWPS can be an effective alternative to securing wheelchairs and scooters in a low $g$ environment. 
The four-point tiedown system was effective at securing wheelchairs, but tended to allow the most displacement of the three systems and allowed significantly more movement than the auto-docking system (avg $12 \mathrm{~mm}$ [0.5 in.] for hard braking, 17 $\mathrm{mm}$ [0.7 in.] for rapid turning). This could have been due to the variability in the tension of the tiedown straps, which was not controlled for during the study.

The unsecured wheelchair trials conducted during this study show the importance of wheelchair securement or containment systems in LATVs. Even during normal driving conditions, an unsecured manual wheelchair experienced an excessive amount of displacement and slid forward in the vehicle when braking downhill. During hard braking and rapid turning trials, all unsecured test wheelchairs and scooters either tipped over or slid across the bus floor. These movements increase the risk of injury to wheelchair users and other near-by passengers (Wolf et al. 2007). This experiment showed that there is a need for wheelchairs to be secured or contained appropriately in LATVs during normal driving conditions, not only to protect the user but also other passengers traveling on LATVs. The unsecured trials also indicate that, as expected, scooters and manual wheelchairs are most likely to move, and a heavier power wheelchair is least likely to move under low acceleration LATV maneuvering.

\section{LATV Accelerations}

LATV accelerations recorded in this study were all less than or equal to $0.75 \mathrm{~g}$. The maximum $g$ levels experienced in this study during normal driving conditions averaged $0.46 \mathrm{~g}$. These values approximate those measured in several previous studies that reported maximum accelerations near $0.40 \mathrm{~g}$ (Rutenberg and Association 2000; Zaworski et al. 2007). The current study recorded a maximum normal driving acceleration of approximately $0.60 \mathrm{~g}$, which is slightly higher than that reported by previous studies (Rutenberg and Association 2000; Zaworski et al. 2007). The type of vehicle and the course traversed during the normal driving trials may explain the difference between accelerations.

During hard braking and rapid turning, the current study reported maximum accelerations of $0.75 \mathrm{~g}$ and $0.56 \mathrm{~g}$, respectively. These values are similar to those found by Zaworski et al. (2007), who reported a maximum of $0.85 \mathrm{~g}$ for braking, and a maximum of $0.39 \mathrm{~g}$ for turning. The slight differences found in the accelerations between studies are most likely due to the difference in the types of buses used (high-floor versus low-floor buses) and the differences in testing procedures. Regardless of the slight differences in the maximum accelerations reported in the literature, all acceleration values remained below $1 \mathrm{~g}$. 


\section{Study Limitations}

This study did not examine the effect of the vehicle-anchored occupant restraint on wheelchair displacement. Vehicle-mounted occupant restraints could provide additional securement, further reducing the displacement of wheelchairs during all driving conditions. The ISO loader gages in this study were restrained to each of the test wheelchairs and could have affected the wheelchair measurements during normal driving, hard braking, and rapid turning trials. In this study, the amount of tension applied to the four-point tiedowns was not measured. The wheelchairs also were secured randomly by various researchers; although this represents real usage of tiedowns, it also may have influenced the magnitude of displacement of the test wheelchairs and scooter when secured by the four-point tiedown system. The ISO loader gages used in this study represent the mass of a 50th percentile male only. They are not representative of how an actual wheelchair-seated individual would respond in low $g$ conditions and how this may influence wheelchair displacement.

This study was not conducted to make recommendations on wheelchair types that are appropriate and safer for use in LATVs. Additional research is needed to better understand how wheelchair type (manual, powered, scooter) and wheelchair securement use affects wheelchair displacement and occupant safety in LATVs.

\section{Conclusions}

All wheelchair securement systems tested in this study met the ADA displacement requirement by limiting wheelchair displacement to less than $51 \mathrm{~mm}$ (2 in.) during normal driving conditions. In addition, all systems met the ADA requirement for wheelchair displacement during hard braking and rapid turning maneuvers. The auto-docking system allowed significantly less displacement than the four-point tiedown system. Accelerations recorded in the LATV remained below $0.76 \mathrm{~g}$, providing further justification, in addition to the low frequency of large impacts reported by Blower et al. (2005), for wheelchair securement performance requirements of $1 \mathrm{~g}$.

New standards specifying methods to test wheelchair containment systems for use in LATVs are presently under development (ISO 10865). Results also indicate that there is a need to secure wheelchairs in LATVs even during normal driving conditions to prevent possible injury to the wheelchair occupant and other vehicle passengers. A follow-up project to this study includes having wheelchair users and LATV operators use and evaluate each wheelchair securement system on an LATV over a predetermined route and give feedback as to their likes and dislikes of each 
system. This feedback will be important for optimizing wheelchair securement systems for independent use by wheelchair seated passengers riding on LATVs.

\section{Acknowledgements}

Support for this study was provided by the Department of Education, National Institute on Disability and Rehabilitation Research and the Rehabilitation Engineering Research Center on Wheelchair Transportation Safety (Grant \#H133E060064). The opinions expressed herein are from the authors and do not necessarily represent those of the funding agency.

The authors express their thanks to Pittsburgh Port Authority Transit for securement system installation and providing a vehicle and drivers for testing purposes. The authors acknowledge BodyPoint, Q'Straint, Invacare, Amigo, Sunrise Medical, and Sure-Lok for their support with designing prototypes, manufacturing components, and donating wheelchairs, securement systems, and seat belts.

\section{References}

ANSI/RESNA. 2001. ANSI/RESNA WC-19: Wheelchairs Used as Seats in Motor Vehicles. Arlington, American National Standards Institute (ANSI)/Rehabilitation Engineering Society of North America (RESNA).

Blower, D., L. Schneider et al. 2005. Characterization of transit-bus accidents resulting in passenger injuries for use in developing alternative methods for transporting wheelchair-seated travelers. International Truck \& Bus Safety \& Security Symposium, Itasca (IL), National Safety Council.

Buning, M., C. Getchell et al. 2007. Riding a bus while seated in a wheelchair: A pilot study of attitudes and behavior regarding safety practices. Assistive Technology 19(4): 166-179.

Foreman, C., J. Hardin et al. 2001. The challenges of wheelchair securement: Searching for solutions. Tampa, FL: National Center for Urban Transportation Research-Center for Transit Research, University of Southern Florida.

Fournier, E. 1997. In-vehicle evaluation of a wheelchair head and back support for use on inter-city buses. Toronto, Ontario, Canada City Province: Biokinetics and Associates, Rhodes \& Associates. 
Frost, K., and G. Bertocci. 2009. Wheelchair securement and occupant restraint practices in large accessible transit vehicles. RESNA, New Orleans.

Hobson, D. A., and L. van Roosmalen. 2007. Towards the next generation of wheelchair securement. Development of a demonstration UDIG-compatible wheelchair docking device. Assistive Technology 19(4): 210-222.

International Standards Organization (1992). ISO7176-Wheelchairs - Part 11: Test dummies. Geneva, Switzerland, International Standards Organization.

International Standards Organization. 2001. ISO7176-Part 19:Technical systems and aids for disabled or handicapped persons-Wheelchairs : Wheeled mobility devices for use in motor vehicles. ISO. Geneva, Switzerland, International Standards Organization.

International Standards Organization (2010). ISO/DIS 10865 Part 1: Assistive products for persons with disability-Wheelchair containment and occupant retention systems for motor vehicles designed for use by both sitting and standing passengers-Part 1: Systems for rearward facing wheelchair-seated passengers. ISO. Geneva, Switzerland, International Standards Organization.

Mercer, P. W., and J. R. Billing. 1990. Assessment of a transportable mobility aid in severe driving conditions - An exploratory test. Ontario, Vehicle Technology Office Transportation Technology and Energy Branch: 1-53.

Rutenberg, U. 1995. Urban transit bus accessibility considerations. Toronto, Ontario, Canada, Canadian Urban Transit Association.

Rutenberg, U. 2000. Accommodating mobility-aids on Canadian low-floor buses using the rear-facing position design: Experience, issues, and requirements. STRP Report 13, November.

Rutenberg, U., R. Baerg et al. 2005. Assessment of low floor transit bus g forces on rear-facing wheelchair securement systems. Montreal, Quebec, Canada, Transportation Development Centre.

Shaw, G. 2008. Investigation of large transit vehicle accidents and establishing appropriate protection for wheelchair riders. Journal of Rehabilitation Research and Development 45(1): 85-108.

Shaw, G., and T. Gillispie. 2003. Appropriate protection for wheelchair riders on public transit buses. Journal of Rehabilitation Research and Development 40(4): 309-320. 
Society of Automotive Engineers. 1993. SAE J2181: Steady-state circular test procedure for trucks and buses. Warrendale, PA, SAE.

Society of Automotive Engineers. 1999. SAE J2249: Wheelchair tiedowns and occupant restraint systems - Surface vehicle recommended practice. Warrendale, PA, SAE.

U.S. Department of Transportation. 2007). Part 38 - Accessibility specifications for transportation vehicles. Title 49, Volume 1. Code of Federal Regulations, Federal Transit Administration.

Wolf, P., and L. van Roosmalen. 2007. Wheelchair tiedown and occupant restraint system issues in the real world and the virtual world: Combining qualitative and quantitative research approaches. Assistive Technology 19(4): 188-196.

Zaworski, U., K. Hunter-Zaworski et al. 2007. Bus dynamics for mobility-aid securement design. Assistive Technology 19(4): 200-209.

\section{About the Authors}

Michael Turkovich, Ph.D. (mjt52@pitt.edu) received his doctoral degree from the University of Pittsburgh Department of Bioengineering and collaborates on projects in the Rehabilitation Engineering Research Center on Wheelchair Transportation Safety.

Linda van Roosmalen, Ph.D. (info@lincdesign.biz) is a Visiting Assistant Professor at the University of Pittsburgh Department of Rehabilitation Science and Technology and Department of Bioengineering. She is one of the lead investigators in the Rehabilitation Engineering Research Center on Wheelchair Transportation Safety and is Principal of LINC Design, LLC.

Douglas A. Hobson, Ph.D. (dhobson1@mac.com) is Emeritus Associate Professor at the University of Pittsburgh in the Department of Rehabilitation Science and Technology.

Erik Porach, B.S. (eap26@pitt.edu) is a Research Specialist at the University of Pittsburgh Department of Rehabilitation Science and Technology. He supports projects in the Rehabilitation Engineering Research Center on TeleRehabilitation, the RERC on Spinal Cord Injury, and the RERC on Wheelchair Transportation Safety. 